\title{
Expression of CD133 and Aldehyde Dehydrogenase 1A1 in Borderline Ovarian Tumor and Their Correlation with International Federation of Gynecology and Obstetrics
}

\author{
Birgitta M. Dewayani*, Hasrayati Agustina, Desi Puspita, Bethy S. Hernowo, Sri Suryanti \\ Department of Anatomic Pathology, Padjadjaran University, Faculty of Medicine, Bandung, Indonesia
}

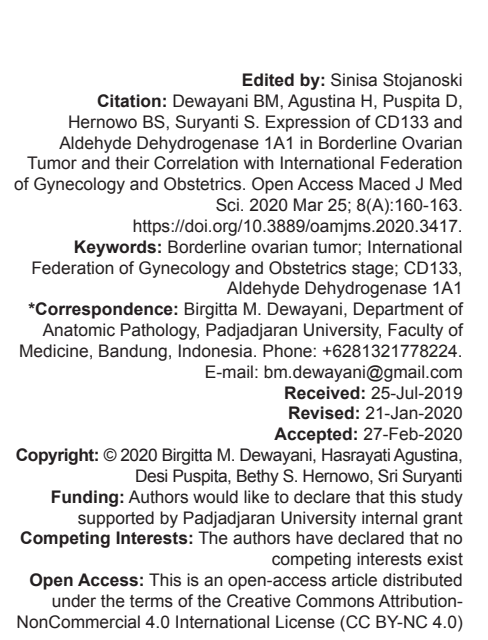

Abstract

BACKGROUND: Borderline ovarian tumor (BOT) is an epithelial ovarian tumor with atypical epithelial proliferation without stromal invasion. BOTs may have an aggressive fashion with associated microinvasion feature, peritoneal "implants," regional lymphadenopathy, and higher International Federation of Gynecology and Obstetrics (FIGO) stage. CD133 is cancer stem cells (CSCs) marker that promotes cell proliferation and tumor invasion through induction of nuclear factor kappa $B$ and upregulation of MMP9. Aldehyde dehydrogenase 1A1 (ALDH1A1) is CSCs marker that promotes cell proliferation through the upregulation of C-MYC and cyclin-D1

AIM: The aim of this study was to investigate the correlation between CD133 and ALDH1A1 expression with FIGO stage. This research was performed as an analytic-observational with a cross-sectional design.

METHODS: This research using a paraffin block of patients diagnosed as BOT in Hasan Sadikin Hospital Bandung. Samples were divided in two groups: FIGO stage IA and FIGO stage >IA. All samples were stained by immunohistochemistry CD133 and ALDH1A1. All data were analyzed using the Chi-square test with a significant level $5 \%$.

RESULTS: This study showed a statistically significant correlation between CD133 $(p=0.047)$ and ALDH1A1 $(p=$ 0.042 ) expression with FIGO stage in BOT. Multivariate analysis showed there was no correlation between CD133 and ALDH1A1 in the affected of FIGO stage in BOT.

CONCLUSION: CD133 and ALDH1A1 in BOT can be considered as a factor to predict the prognosis of BOT through predict FIGO stage.

\section{Introduction}

Epithelial ovarian tumor (EOT) consists of benign EOT (cystadenoma), malignant EOT (carcinoma), and borderline ovarian tumor (BOT) [1]. Characteristics of BOTs are atypical epithelial proliferation and without stromal invasion [1], [2], [3]. BOTs comprise up to $15-20 \%$ of ovarian epithelial neoplasms. BOTs often occurred in younger women with an average age is 40 years old [2]. According to the WHO classification, BOT consists of serous BOT, mucinous BOT, endometrioid BOT, and borderline Brenner tumor [1]. BOT often occurred at an earlier stage and has a better prognosis than malignant ovarian tumors. Some BOTs may have an aggressive fashion with associated peritoneal "implants," regional lymphadenopathy, and higher International Federation of Gynecology and Obstetrics (FIGO) stage [1], [4].

Cancer stem cells (CSCs) are a small proportion of tumor cells which are proposed to be able to proliferate and self-renew and invasion extensively [5]. CD133 and aldehyde dehydrogenase 1A1 (ALDH1A1) are CSC marker that can identify in ovarian tumor and correlate with a stage in ovarian cancer [6]. CD133 promotes cell proliferation, tumorigenesis, and tumor invasion and metastasis through induction of nuclear factor kappa B and upregulation of MMP9 [7]. ALDH1A1 promotes cell proliferation through upregulation of c-MYC and cycin-D1 [8]. High tumor cell proliferation, high ability of invasion, implants, and involvement of regional lymphadenopathy in BOT may influence FIGO stage and few cases of recurrences may occur [1]. Stage in BOT currently based on FIGO staging system. FIGO staging system according to involvement of tumor mass, whether unilateral or bilateral, tubal involvement, tumor mass in ascites fluid, peritoneal, lymph node involvement, or distant metastasis [9]. Loizzi et al. found that majority cases BOT had FIGO stage I $(85.5 \%)$ and the remainder exhibited FIGO stage II (7.3\%) and FIGO stage III (7.3\%) [3].

To the best of our knowledge, no data have been reported until now about the role of the immunohistochemically assessed expression of CD133 and ALDH1A1 of BOT in a large, single Institution series in Indonesia. 


\section{Subjects and Methods}

This study uses an analytic observational method with a cross-sectional study design and retrospective data retrieval/collection. Ethical clearance has been approved/assessed by Health Research Ethic Commission, Padjadjaran University, assessment number 1144/UN6.KEP/EC/2018. The inclusion criteria: The samples were obtained from patients registered at Hasan Sadikin Hospital, were diagnosed with BOT (serous BOT, mucinous BOT, endometrioid BOT, and borderline Brenner tumor) during January 2013 to July 2018. All paraffin blocks from all samples were good and all samples have complete data about FIGO stage in the medical record.

All samples were classified as FIGO stage IA (unilateral involvement) and FIGO stage $>$ IA (bilateral involvement or involvement to another organ). Analysis of expression: Samples from paraffin blocks were prepared for immunohistochemistry (IHC) analysis; IHC analysis was performed based on the protocol provided by the anatomical pathology laboratory. The slides were visualized under the microscope with three reviewers that have no knowledge about clinicopathologic data.

\section{Histoscore calculation}

The positive result was shown/visualized as brown staining on the tumor cell. Analysis on CD133 was evaluated by brown staining in the cytoplasmic membrane of tumor cells and ALDH1A1 expression was evaluated by brown staining assessed in the cytoplasm. The stain intensity and distribution measured under the microscope was then converted into histoscore and categorized in high and low expression. This study used antibody CD133 (Polyclonal antibody, Elabscience) with dilution 1:200 and antibodyALDH1A1 (Polyclonal antibody, Santacruz) with dilution 1:800. The intensity was scored as having no expression (0), or weak (1), moderate (2), or strong (3) tumor-cell staining. Distribution was scored as having no expression (0), or $<10 \%$ (1), $10-50 \%$ (2), or $>50 \%$ of (3) tumor-cell staining.

Histoscores obtained from the samples were represented on a scale of 0-9. Histoscores were classifying to weak (histoscore $\leq 6$ ) and strong (histoscore $>6$ ).

This research was performed as an analyticobservational with a cross-sectional design. Statistical analysis was performed with SPSS using the Chisquare test with a significant level $5 \%$. $p<0.05$ was considered to be statistically significant and in the test of independence (multivariate analysis), a $p>0.05$ was considered to show independence between CD133 and ALDH1A1.

\section{Results}

During January 2013-July 2018, there were 101 cases of BOT which were registered; however, only 84 samples were matched to inclusion criteria. Age ranging from 18 to 71 years (median 42 years) and 47 patients (56\%) was over 40 years of age. The most common histopathology type was mucinous BOT in 59 patients $(70.2 \%)$ and followed by serous BOT in 22 patients $(26.2 \%)$ (Table 1$)$.

Table 1: Characteristic of research subject

\begin{tabular}{ll}
\hline Variable & $\mathrm{n}=84(\%)$ \\
\hline Age (year) & \\
Mean \pm Std. & $41.13 \pm 13.025$ \\
Median & 42.00 \\
Range (min-max) & $18.00-71.00$ \\
$<40$ & $37(44.0 \%)$ \\
$\geq 40$ & $47(56.0 \%)$ \\
Type of histopathology & \\
Mucinous BOT & $59(70.2 \%)$ \\
Serous BOT & $22(26.2 \%)$ \\
Endometrioid BOT & $2(2.4 \%)$ \\
Borderline Brenner tumor & $1(1.2 \%)$ \\
Parity & $15(17.9 \%)$ \\
P0 & $69(82.1 \%)$ \\
P $\geq 1$ &
\end{tabular}

From this study, we found 66 patients with FIGO stage IA. Fourteen patients (21.2\%) showed low expression of CD133 and 52 cases (78.8\%) showed high expression of CD133 (Figure 1a). Moreover, 18 patients with FIGO stage more than IA showed low expression in 8 cases $(44.4 \%)$ and high expression in 10 cases (55.6\%). From statistical analysis showed the significant correlation between CD133 ( $p<0.05$ ) expression with FIGO stage in BOT. Expression of ALDH1A1 in FIGO stage IA showed low expression in 11 patients $(16.7 \%)$ and high expression in 55 patients $(83.3 \%)$ (Figure 1b). Patients with FIGO stage >IA showed low expression in 7 patients $(38.9 \%)$ and high expression in 11 patients (61.1\%). From statistical analysis showed the significant correlation between ALDH1A1 $(p<0.05)$ expression with FIGO stage in BOT (Table 2). Multivariate analysis showed there was no correlation between CD133 and ALDH1A1 in the affected of FIGO stage in BOT (Table 3).

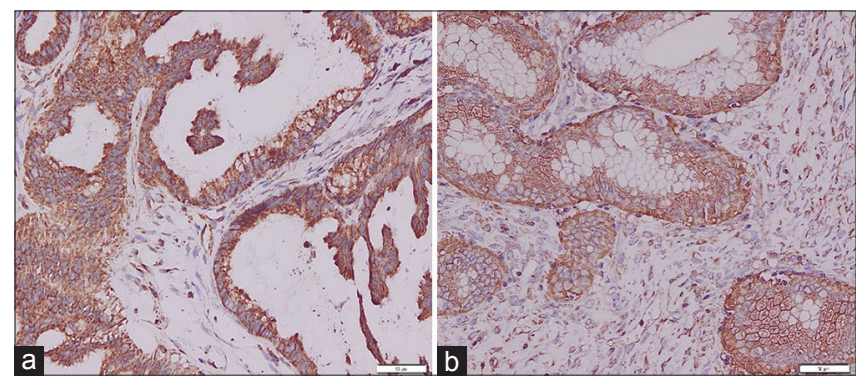

Figure 1: CD133 and aldehyde dehydrogenase 1A1 (ALDH1A1) expression of borderline ovarian tumor in this study. (a) CD133 strong expression in this study, tumor cell stained with CD133 antibody in the cytoplasmic membrane (200×). (b) ALDH1A1 strong expression in this study, tumor cell stained with ALDH1A1 antibody in the cytoplasm (200x 
Table 2: CD133 and ALDH1A1data on FIGO stage of BOT group

\begin{tabular}{|c|c|c|c|}
\hline \multirow[t]{2}{*}{ Variable } & \multicolumn{2}{|c|}{ FIGO stage of BOT } & \multirow[t]{2}{*}{$\mathrm{p}$ value } \\
\hline & $\mathrm{IA}(\mathrm{n}=66) \%$ & $>\mid A(n=18) \%$ & \\
\hline Histoscore CD133 & & & $0.047^{\star \star}$ \\
\hline Low & $14(21.2)$ & $8(44.4)$ & \\
\hline High & $52(78.8)$ & $10(55.6)$ & \\
\hline Histoscore ALDH1A1 & & & $0.042^{\star \star}$ \\
\hline Low & $11(16.7)$ & $7(38.9)$ & \\
\hline High & $55(83.3)$ & 11(61.1) & \\
\hline
\end{tabular}

\section{Discussion}

Prognostic factors in BOT include age, FIGO stage, residual disease following surgery, type of peritoneal implants (with or without invasion), presence of microinvasion, micropapillary pattern, and the CA-125 value. Prognosis of BOT overall has a positive prognosis because over $80 \%$ of cases are diagnosed at an early stage of the disease and radical surgery and surgical staging are the standard of care for this disease includes hysterectomy, bilateral salpingo-oophorectomy, omentectomy, multiple biopsies, and peritoneal cytology [2].

Table 3: Multivariate analysis correlation CD133 and ALDH1A1 expression with FIGO stage of BOT

\begin{tabular}{|c|c|c|c|}
\hline Variable & Estimation rate & SE & $p$ value \\
\hline CD133 & -0.972 & 0.577 & 0.092 \\
\hline ALDH1A1 & -1.035 & 0.602 & 0.602 \\
\hline Constant & 2.128 & 1.354 & 0.116 \\
\hline
\end{tabular}

FIGO stage for ovarian carcinoma includes BOT which is the most superior staging system for predict patient prognosis through discriminating survival outcomes [10]. FIGO stage is according to the involvement of tumor mass, whether unilateral or bilateral, tubal involvement, tumor mass in ascites fluid, peritoneal, lymph node involvement, or distant metastasis [9]. The primary route of ovarian cancer expanding/metastasis includes intraperitoneal implantation of exfoliated tumor cells and spreading through retroperitoneal lymphatic channels [10].

CSCs are a small proportion of tumor cells which are proposed to be able to proliferate and selfrenew and invasion extensively [5]. CD133 is one of CSCs marker in ovarian cancer [6]. Long et al. found that the presence of CD133+ ovarian CSC can undergo an epithelial-mesenchymal transition-like process and display enhanced metastatic capacity of tumor cells in vitro and in vivo through secreting soluble mediators. CCL5 is chemokine which is one of the soluble mediators produced by CD133+ ovarian CSC. Signaling of CCL5 is an important thing for the invasive capability of CSC and tumor cells [11].

This study performed CD133 expression analysis on FIGO stage and microinvasion feature of BOT. The result from IHC staining confirms that there are $55.6 \%$ of patients with FIGO stage more than IA showed high expression of CD133. From statistical analysis showed the significant correlation between
CD133 $(p<0.047)$ expression with FIGO stage in BOT. High expression of CD133 results in high stage FIGO stage (FIGO stage more than IA).

ALDH1A1 is a marker of CSC that promotes self-renewal, differentiation, and self-protection of cells. High expressions of ALDH1A1 correlated with poor cancer prognosis but not correlate with highly malignant phenotypes [8]. Hence, in this study, we performed ALDH1A1 expression analysis on FIGO stage of BOT. The result from $\mathrm{IHC}$ staining confirms that there are $61.1 \%$ of patients with FIGO stage more than IA showed high expression of ALDH1A1. From statistical analysis showed the significant correlation between ALDH1A1 $(p<0.042)$ expression with FIGO stage in BOT. High expression of ALDH1A1 results in high stage FIGO stage (FIGO stage more than IA).

This study also performed multivariate analysis correlation CD133 and ALDH1A1 expression with FIGO staging. There was no correlation between CD133 and ALDH1A1 expression with FIGO stage.

\section{Conclusion}

There was a significant correlation between CD133 with FIGO staging and correlation between ALDH1A1 with FIGO stage.

\section{References}

1. Kurman RJ, Carcangiu ML, Herrington CS, Young $\mathrm{RH}$. WHO Classification of Tumours of Female Reproductive Organs. $4^{\text {th }}$ ed. Lyon: International Agency for Research on Cancer; 2014. p. 15-39.

2. Patrono MG, Minig L, Diaz-Padilla I, Romero N, Moreno JF, Garcia-Donas J. Borderline tumours of the ovary, current controversies regarding their diagnosis and treatment. Ecancermedicalscience. 2013;7:379.

PMid:24386008

3. Loizzi V, Selvaggi L, Leone L, Latorre D, Scrdigno D, Magazzino $\mathrm{F}$, et al. Borderline epithelial tumors of the ovary: Experience of 55 patients. Oncol Lett. 2015;9(2):912-4. https:// doi.org/10.3892/ol.2014.2758 PMid:25621067

4. Lalwani N, Shanbhogue AK, Vikram R, Nagar A, Jagirdar J, Prasad SR. Current update on borderline ovarian neoplasms. AJR. 2010;194(2):330-6. https://doi.org/10.2214/ajr.09.3936 PMid:20093592

5. Ferrandina G, Martinelli E, Petrillo M, Prisco MG, Zannoni G, Sioletic S, etal. CD133 antigen expression in ovarian cancer. BMC Cancer. 2009;9:221. https://doi.org/10.1186/1471-2407-9-221 PMid:19583859

6. Tao Y, Li H, Huang $\mathrm{R}$, Mo D, Zeng $\mathrm{T}$, Fang $\mathrm{M}$, et al Clinicopathological and prognostc significance of cancer stem cell markers in ovarian cancer patients: Evidence from 52 studies. Cell Physiol Biochem. 2018;46(6):1716-26. https://doi. 
org/10.1159/000489586

PMid:29730663

7. Li Z. CD133: A stem cell biomarker and beyond. Exp Hematol Oncol. 2013;2(1):17.

PMid:23815814

8. Tomita H, Tanaka K, Tanaka T, Hara A. Aldehyde dehydrogenase 1A1 in stem cells and cancer. Oncotarget. 2016;7(10):11018-27. https://doi.org/10.18632/oncotarget.6920 PMid:26783961

9. Javadi S, Ganeshan D, Qayyum A, Iyer R, Bhosale P. Ovarian cancer, the revised FIGO staging system, and the role of imaging. AJR Am J Roentgenol. 2016;206(6):1351-60. https:// doi.org/10.2214/ajr.15.15199
PMid:27042752

10. Suh DH, Kim TH, Kim JW, Kim SY, Kim HS, Lee TS, et al. Improvements to the FIGO staging for ovarian cancer: Reconsideration of lymphatic spread and intraoperative tumor rupture. J Gynecol Oncol. 2013;24(4):352-358. https://doi. org/10.3802/jgo.2013.24.4.352 PMid:24167671

11. Long H, Xiang T, Qi W, Huang J, Chen J, He L, et al. CD133+ ovarian cancer stem-like cells promote non-stem cancer cell metastasis via CCL5 induced epithelial-mesenchymal transition. Oncotarget. 2015;6(8):5846-59. https://doi.org/10.18632/ oncotarget.3462

PMid:25788271 